\title{
CITATION OF THE SCIENTIFIC PRODUCTIVITY OF CHEMISTS IN CHILE
}

\author{
BERNABÉ L. RIVAS* AND DANIEL A. PALACIO \\ Polymer Department, Faculty of Chemistry, University of Concepcion, Casilla 160-C, Concepcion, Chile.
}

\begin{abstract}
It is very relevant give the scientific productivity to the world scientific community as well as to the society. One of the most common and important media is through published papers in Journals recognized for different international sources and also measured by the impact factor. One the last factor has been the Hirsh index or H-index. The current contribution gives information of the chemists in Chile with $H$-index higher or equal 20 with respect to the self- citation (T) according to the recent publication.
\end{abstract}

Keywords: Self-citations, $H$-index, Chemists, Chile.

\section{INTRODUCTION}

For much of human history, however, science had little impact on people's daily lives. Scientific knowledge was developed by a few, with the sole desire to increase knowledge, its dissemination was done in very small circuits and had no greater practical applications. It was only at the beginning of the eighteenth century, with the advent of the Industrial Revolution, that the stage began to change radically, as an explosion of scientific and technological applications detonated that has since accelerated without pause. Today, the lives of the vast majority of people are deeply influenced by science and technology, in virtually every sphere imaginable. Science and technology have given support to enormous power to transform the world, accommodating it to its best.

Countries have been learning that finding such solutions is a long and complex process, and that it often involves investing large resources. To successfully approach this process, we need knowledge in different disciplines, which is traditionally generated by scientific researchers, universities, public centers or companies. Scientific research, therefore, has a fundamental relevance for all countries, and Chile is no exception. Chile has seen significant progress in recent decades in terms of scientific development. Today we have more researchers, various programs to fund research activities, and the world today offers more advanced tools to experimentally address certain questions, allowing to generate more and better knowledge. However, the scale and complexity of the challenges is increasing, and therefore, it is necessary not only to maintain, but to enhance our capacities in terms of scientific development and innovation. Science has an important role to play in solving our problems and challenges, and that is why state support is critical, and the participation of companies and other private sector actors is also of enormous relevance.

During the 1920 s, science gradually began to abandon its merely utilitarian sense to transform into scientifically-based theoretical disciplines. During this century the sciences were epistemologically divided between exact sciences and applied sciences. As exact sciences were understood those who based their work on experimentation and observation, systematizing their knowledge in mathematical language. These include mathematics, physics, chemistry, astronomy and some branches of biology such as biochemistry. On the other hand, towards the second half of the twentieth century emerged the concept of applied sciences, a denomination that grouped those disciplines that specialized in solving practical problems, using other sciences such as the exact ones. These sciences focused their work on the development of technology and industry. In this group are the economy, aeronautics, computer science, engineering, electrical, between other.

According to that, the development of the sciences experienced in Chile was halted by the effects of the economic crisis in the 1930s, a financial debacle that involved directing economic efforts to other objectives. This event also increased for a time the isolation of the scientific centers of Chile in relation to those of the rest of the world. However, it was not only the economic crisis that affected the development of the exact sciences in Chile. Throughout the twentieth century, international contingency directly influenced the development of the exact sciences. From the 1960s onwards, the exact sciences in Chile clearly defined their borders, differing not only within the curricula of universities, but also through the foundation of specific faculties, already from the engineering and research centers operated by them. In the following was the creation of specialized scientific societies. For its part, the State consolidated its leading role as the main promoter of national sciences through the founding of the Academy of Sciences in 1964, the creation of the National Commission for Scientific and Technological Research (CONICYT) in 1967, from the last year National Agency for Research and Development (ANID). Thus, during the twentieth century, and from the action of the State, the sciences were considered not only as a useful set of tools for the progress and development of the nation, but also as disciplines of theoretical research and creation of new technologies. This allowed to significantly increase both the quality and the number of researchers and scientists dedicated to this area. On the other hand, he ended the work of isolated individuals and established an active community of professionals prone to debate and discuss. To respond more efficiently to the new needs of our country, from the new millennium the sciences have been inextricably linked with technological development, focusing its work towards innovation and linking with international networks of work and research and innovation ${ }^{1}$.

Accordingly with the development of the Chilean science, the chemistry has been very strong support to the growing and development of the Chilean science. From the 60 decade and particularly during the seventeen decade with the starting and development of Graduate Programs from the three older Universities, Universidad de Chile (UCh), Pontificia Universidad Catolica de Chile (PUC), and Universidad de Concepcion (UdeC). Subsequently, started other two programs at the Universidad de Santiago de Chile (USACH), and Pontificia Universidad Catolica de Valparaiso (PUCV). These Graduate Programs on Chemistry are been fundamental for the scientific growing of the chemistry. Therefore, practically almost the scientific activity is carried at the Universities, no more $10 \%$ is performed at the industries. Due to that the almost of the scientific productivity, through published papers is coming from the Universities. Therefore, the innovation also shows up to now a little production measured by products like patents.

Scientific productivity at the global level plays an important role in the visibility of institutions, countries, and especially in the recognition of researchers, which is why it is often more recurrent to judge by the number of companies received the work of each researcher ${ }^{2}$. On the other hand, the most of Chilean Universities include for the academic career the performing of research, and the scientific disciplines as chemistry, published papers. In principle was considered the number of papers, subsequently was considered the impact factor of the Journal, and currently the $H$-index which is defined as follows"A scientist has index $h$ if $h$ of his or her $N_{p}$ papers have at least $h$ citations each and the other $\left(N_{p}-h\right)$ papers have $\leq h$ citations each"3,4, and citation numbers. Defining the H-index as a standard to measure academic performance, although its introduction as a tool to assess impact the researchers raised some concerns in the scientific community ${ }^{5}$. Nevertheless, not only in Chile, but around the word, it is not possible discard completely the effect of the self-citations of the scientists. Currently, different studies have been carried out to help determine the citation ranking of academic researchers, where different methods have been used to classify researchers in the academic world ${ }^{6-8}$.

These methods are based on bibliometric indicators such as citations, offering a perspective beyond the scientific impact that researchers may have in dealing with self-citations, that role played by authors' self-citations in the scientific community presents a discussion continues in the biometric literature ${ }^{9-11}$. Therefore, it is very important the recent publication on the self-citation test, $\mathrm{T}$, which considers the $H$-index and the total citations. It is shown that the $H$-index squared divided by the number of citations predicts self-citations. 
The test is simple to apply based on Google Scholar author profiles. Bibliometric data for more than 100,000 researchers worldwide were used to assess the proposed test. Test values of 0.35 or more indicate high ratios of selfcitation while test values below 0.2 suggest low ratios of self-citations ${ }^{12}$.

The issue of self-citations has received much attention in the academic community ${ }^{13}$. Self-citations are often perceived negatively as they can result in misleading impressions of a researcher's impact. The debate becomes especially heated when publication metrics are used as incentives ${ }^{12,14}$. However, there is a diversity of opinions in the scientific society regarding this topic that are increasing, because some researchers believe that self-citations is a way of artificially inflating in $\mathrm{H}$-index. Moreover, the bibliometricians consider it partly reasonable that self-citations are a natural part of the scientific procedure ${ }^{9,15}$.

\section{THE SELF-CITATION TEST}

The proposed test uses the H-index and the total number of citations as provided by Google Scholar author profiles. The proposed test for self-citation is defined as follows ${ }^{12}$ :

$$
T=\frac{(H-\text { index })^{2}}{\text { Citations }}
$$

The test value $T$ is a value in the range between 0 and 1 . A high value indicates that the author has many self-citations, while a low value indicates few selfcitations.

\section{ANALYSIS OF CHEMISTS IN CHILE}

According to the self-citations defined previously, was defined a sample of chemists in Chile with $H$ - index $\geq 20$ which was arbitrary decided, for a total of 52 researchers which represent around the $10 \%$ of the researchers in chemistry. All the information on $H$-index and citations was obtained from available Index of Journals of Web of Science Core Collection (Science Citation Index Expandex (SCI Expanded 1975-present) and is summarized in Table 1. This information was used as it is available, without further treatment. Through this WEB of Science source is more easy get cites and $\mathrm{H}$-index for each researcher and achieve the corresponding profile. It is possible that this information has a possible mistake on the total, cites but it would occur for all the researchers.

Table 1. Self-citation values for Chemists in Chile.

\begin{tabular}{|c|c|c|c|c|c|c|}
\hline Surname & Name & Affiliation & Citation* & Paper No* & H-index* & $\mathbf{T}$ \\
\hline Arratia & Ramiro & \begin{tabular}{|l} 
Universidad Andres Bello \\
\end{tabular} & 2,222 & 202 & 25 & 0.28 \\
\hline Bollo & Soledad & Universidad de Chile & 1,363 & 63 & 21 & 0.32 \\
\hline Caballero & Julio & Universidad de Talca & 2,594 & 140 & 30 & 0.35 \\
\hline Cardenas-Jiron & Gloria & Universidad de Santiago de Chile & 1,415 & 80 & 21 & 0.31 \\
\hline Carrillo & David & Pontificia Universidad Catolica de Valparaiso & 1,413 & 88 & 21 & 0.31 \\
\hline Cassels & Bruce $\mathrm{K}$. & Universidad de Chile & 3,066 & 179 & 29 & 0.27 \\
\hline Castro & Enrique A. & Pontificia Universidad Catolica de Chile & 4,304 & 119 & 40 & 0.37 \\
\hline Chamorro & Eduardo & Universidad Andres Bello & 2,869 & 81 & 28 & 0.27 \\
\hline Contreras & Renato & Universidad de Chile & 6,953 & 199 & 34 & 0.17 \\
\hline Cordova & Ricardo & Pontificia Universidad Catolica de Valparaiso & 1,595 & 88 & 24 & 0.36 \\
\hline Costamagna & Juan & \begin{tabular}{|l|} 
Universidad de Santiago de Chile \\
\end{tabular} & 2,058 & 144 & 23 & 0.26 \\
\hline Diaz & Fernando R. & Pontificia Universidad Catolica de Chile & 1,493 & 135 & 20 & 0.27 \\
\hline Encinas & Maria & \begin{tabular}{|l|} 
Universidad de Santiago de Chile \\
\end{tabular} & 4,077 & 152 & 32 & 0.25 \\
\hline Escalona & Nestor & \begin{tabular}{|l} 
Pontificia Universidad Catolica de Chile \\
\end{tabular} & 1,761 & 98 & 25 & 0.35 \\
\hline Gargallo & Ligia & Universidad de Tarapaca & 2,471 & 268 & 24 & 0.23 \\
\hline Garland & Maria T. & Universidad de Chile & 3,652 & 305 & 29 & 0.23 \\
\hline Gautier & Juan L. & Universidad de Santiago de Chile & 2,612 & 139 & 26 & 0.26 \\
\hline Isaacs & Mauricio & Pontificia Universidad Catolica de Chile & 1,336 & 76 & 21 & 0.33 \\
\hline Jaque & Pablo & Universidad de Chile & 1,698 & 62 & 24 & 0.34 \\
\hline Aguirre & Maria J. & Universidad de Santiago de Chile & 1,924 & 114 & 24 & 0.30 \\
\hline Lissi & Eduardo & Universidad de Santiago de Chile & 11,735 & 521 & 48 & 0.20 \\
\hline Mansilla & Hector D. & \begin{tabular}{|l|} 
Universidad de Concepcion \\
\end{tabular} & 4,359 & 124 & 35 & 0.28 \\
\hline Manzur & Carolina & Pontificia Universidad Catolica de Valparaiso & 1,264 & 81 & 21 & 0.35 \\
\hline Matsuhiro & Betty & \begin{tabular}{|l|} 
Universidad de Santiago de Chile \\
\end{tabular} & 2,336 & 108 & 26 & 0.29 \\
\hline Maya & Juan D. & Universidad de Chile & 2,586 & 117 & 29 & 0.33 \\
\hline Moreno-Villoslada & Ignacio & Universidad Austral de Chile & 1,598 & 86 & 22 & 0.30 \\
\hline Niemeyer & Hermann M. & Universidad de Chile & 5,947 & 271 & 37 & 0.23 \\
\hline Olea-Azar & Claudio & Universidad de Chile & 2,538 & 121 & 31 & 0.38 \\
\hline Pecchi & Gina & Universidad de Concepcion & 2,018 & 103 & 27 & 0.36 \\
\hline Pérez & Patricia & Universidad Andrées Bello & 7,955 & 123 & 40 & 0.20 \\
\hline Pooley & Sonia A. & Universidad de Concepcion & 1,040 & 67 & 20 & 0.38 \\
\hline Quijada & Raul & Universidad de Chile & 3,458 & 156 & 31 & 0.28 \\
\hline Radic & Deodato & Pontificia Universidad Catolica de Chile & 2,171 & 226 & 21 & 0.20 \\
\hline Reyes & Patricio & Universidad de Concepcion & 2,298 & 124 & 30 & 0.39 \\
\hline Richter & Pablo & Universidad de Chile & 1,786 & 104 & 25 & 0.35 \\
\hline Rivas & Bernabe L. & Universidad de Concepcion & 6,587 & 455 & 38 & 0.22 \\
\hline San Martin & Aurelio & Universidad de Magallanes & 1,337 & 105 & 20 & 0.30 \\
\hline Santos & Jose G. & Pontificia Universidad Catolica de Chile & 3,120 & 130 & 36 & 0.42 \\
\hline Schmeda & Guillermo & Universidad de Talca & 4,728 & 221 & 36 & 0.27 \\
\hline Schrebler & Ricardo & Pontificia Universidad Catolica de Valparaiso & 2,280 & 117 & 26 & 0.30 \\
\hline Silva & Leonardo & Universidad de Talca & 3,145 & 133 & 29 & 0.27 \\
\hline Speisky & Hernan & Universidad de Chile & 3,079 & 109 & 31 & 0,31 \\
\hline Spodine & Evgenia & Universidad de Chile & 1,354 & 167 & 23 & 0.39 \\
\hline Squella & Juan A. & Universidad de Chile & 3,381 & 232 & 30 & 0.27 \\
\hline Suwalsky & Mario & \begin{tabular}{|l|} 
Universidad de Concepcion \\
\end{tabular} & 2,108 & 159 & 24 & 0.27 \\
\hline Tapia & Ricardo A. & Pontificia Universidad Catolica de Chile & 1,717 & 117 & 22 & 0.28 \\
\hline Theoduloz & Cristina & Universidad de Talca & 2,352 & 123 & 26 & 0.29 \\
\hline Tiznado & William & \begin{tabular}{|l} 
Universidad Andres Bello \\
\end{tabular} & 1,358 & 75 & 21 & 0.32 \\
\hline Toro & Alejandro & Pontificia Universidad Catolica de Chile & 7,219 & 222 & 42 & 0.24 \\
\hline Ureta & Maria $\mathrm{S}$. & Universidad de Santiago de Chile & 1,289 & 58 & 22 & 0.38 \\
\hline Valderrama & Jose $\mathrm{O}$. & Universidad de Antofagasta & 2,873 & 113 & 24 & 0.20 \\
\hline Zagal & José $\mathrm{H}$. & Universidad de Santiago de Chile & 6,035 & 183 & 43 & 0.31 \\
\hline
\end{tabular}

*Information collected from Web of Science until July 20, 2020. 
This table summarizes the information of 52 researchers in chemistry which is around the $10 \%$ of all chemists in Chile. According to that is possible conclude that the most of them have a large and fruitful scientific productivity. Only some of them correspond to young chemists. A $25 \%$ (13 chemists) of the researchers have lower 100 papers, $71 \%$ (37 chemists) above 200 papers, and $4.0 \%$ (2 chemists) higher 450 papers.

Respect to cites it is relevant that the most of the chemists have between 2,001 and 4,000 cites $(44.2 \%$ ) and $11.6 \%$ higher 6,001 cites (see figure 1 ).

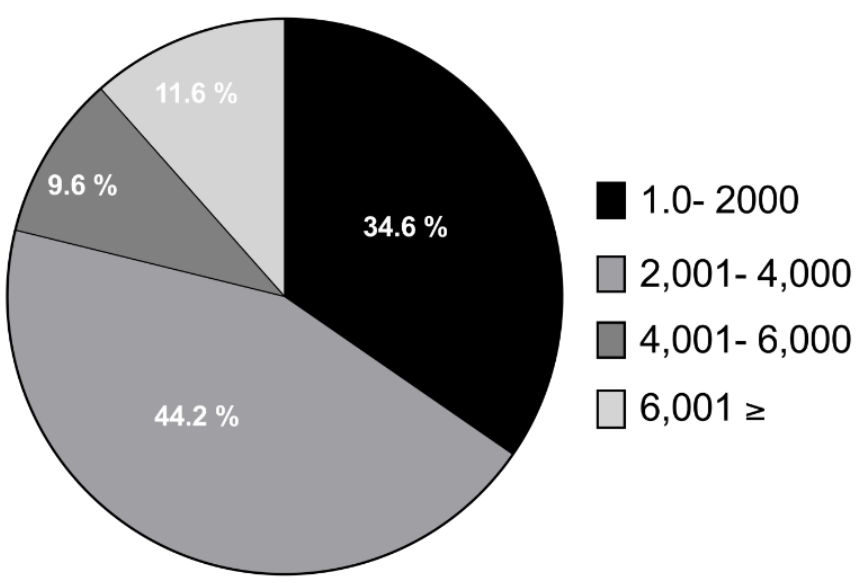

Figure 1. Percentage of researchers in chemistry according to the range of number of citations.

Considering the $H$-index, the most of the researchers have $H$-index between 20.0 and 25.0 ( 24.0 chemists) and above $H$ - index 35.0 ( 9 chemists), highlighting 3 of them with $H$-index above 40.0 which is a great relevance in the Chilean science (see figure 2).

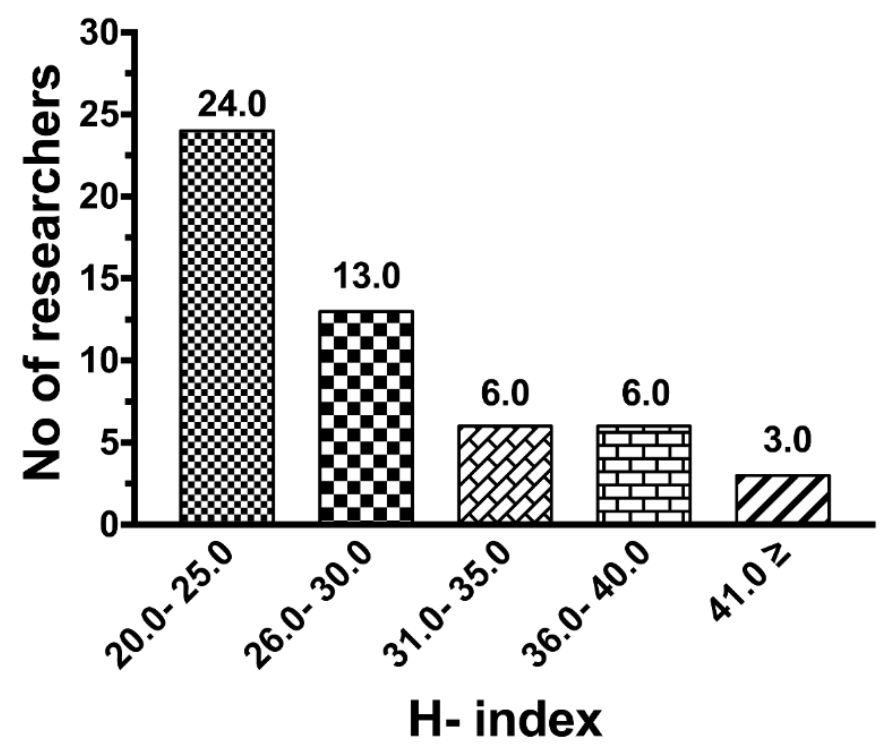

Figure 2. Number of researchers according to $H$-index rank.

This information with a sample of 52 researchers was also treated by quartiles Q1 to Q3, where Q1 indicates that $25 \%$ of the chemists shows an $H$-index $\geq 23$, Q2 shows a $50 \%$ of the chemists presents an $H$-index $\geq 26$, and finally Q3 demonstrates that $75 \%$ of chemists have an $H$-index $\geq 31$.

Finally, considering this new index T, self-citation for the 52 chemists, it is very important that all chemists included in this study show a $\mathrm{T}$ value lower than that 0.43 , the lowest is 0.17 , and the most have a $\mathrm{T}$ value lower 0.30 , and which means that the papers have not a strong effect of the self-citations. A $10 \%$ of the chemists has $\mathrm{T}$ values bellow 0.2 which suggest low ratios of self-citations, and also a $17 \%$ has $\mathrm{T}$ values higher 0.35 indicating high ratios of self-citations (see figure 3).

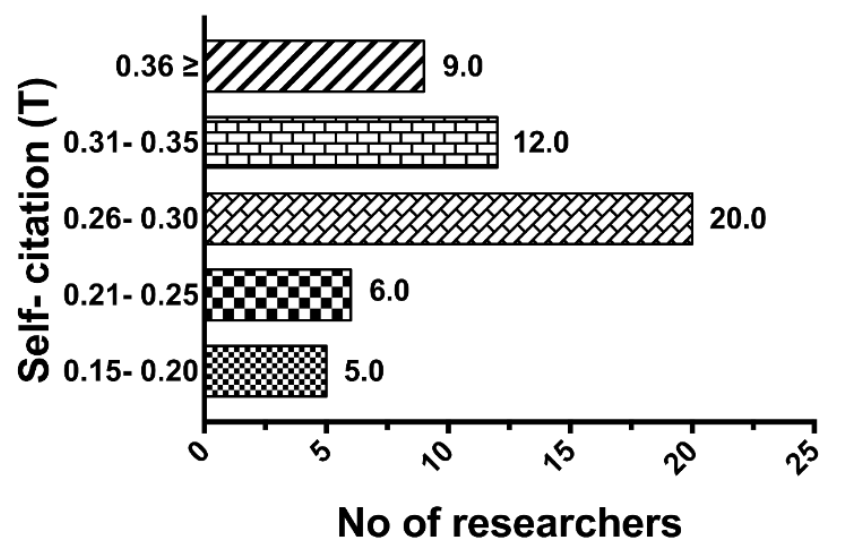

Figure 3. Number of researchers according to self-citation range (T).

\section{CONCLUSIONS}

A simple test for strategic self-citations without self-citation information was informed and analyzed for a group of chemists in Chile with $H$-index $\geq 20$.

Data showed that the test is successful in identifying high levels of selfcitations and that test values of 0.35 or more are contenders for further analysis. According to the information, 43 chemists in Chile (75\%) $(82.6 \%)$ of the total study sample have a self-citation $\mathrm{T}$ value lower or equal to 0.35 which means a little impact of self-citations. It is really important considering also the lowest $\mathrm{T}$ value of 0.17

\section{ACKNOWLEDGEMENTS:}

The authors thank to Mrs. Vania Luksic from Vicerrectoria de Investigación y Desarrollo de la Universidad de Concepcion for her important support on Web of Science information.

\section{REFERENCES}

1. Alvarez, P.; Arellano, A.; Ibánez, P.; Moreno, D.; Mujica, F.; Piedras, J.; Tromben, C.; Urzúa, C.; Balcells, M. Conicyt 2010.

2. Shehatta, I.; Al-Rubaish, A. M. Scientometrics 2019, 120, 775

3. Hirsch, J. E. PNAS 2005, 102, 16569.

4. Kinney, A. L. PNAS 2007, 104, 17943.

5. Ferrara, E.; Romero, A. E. Journal of the American Society for Information Science and Technology 2013, 64, 2332.

6. Amjad, T.; Daud, A.; Aljohani, N. R. Library Hi Tech 2018, 36, 97.

7. Zhao, F.; Zhang, Y.; Lu, J.; Shai, O. Scientometrics 2019, 118, 1119.

8. Dunaiski, M.; Geldenhuys, J.; Visser, W. Journal of Informetrics 2018, 12, 679.

9. Amjad, T.; Rehmat, Y.; Daud, A.; Abbasi, R. A. Scientometrics 2020, 122 915.

10. Bu, Y.; Ding, Y.; Xu, J.; Liang, X.; Gao, G.; Zhao, Y. Journal of the Association for Information Science and Technology 2018, 69, 87.

11. Glänzel, W.; Debackere, K.; Thijs, B.; Schubert, A. Scientometrics 2006, 67, 263.

12. Sandnes, F. E. Scientometrics 2020, 124, 1685

13. Van Noorden, R.; Singh Chawla, D. Nature 2019, 572, 578.

14. Haugen, K. K.; Sandnes, F. E. Scientometrics 2016, 109, 1299.

15. Wolfgang, G.; Bart, T.; Balázs, S. Scientometrics 2004, 59, 63. 